\title{
Effect of total dissolved gas supersaturation on the tolerance of grass carp (Ctenopharyngodon idellus)
}

\author{
Zequn Fan ${ }^{1 \dagger}$, Yuxuan Deng ${ }^{1 \dagger}$, Quan Yuan ${ }^{3}$, Xiaoqing Liu ${ }^{1,2^{*}}$, Haoran Shi ${ }^{1^{*}}$, Cuixia Feng ${ }^{1}$, Yao Yang ${ }^{1}$ \\ and Liangfang $\mathrm{Xu}{ }^{1}$
}

\begin{abstract}
Background: Total dissolved gas (TDG) caused by the rapid outflow of water from dams may threaten the survival of fish. However, few studies have assessed the impact of fish size on their tolerance to TDG supersaturation in the Yangtze River. To investigate the influences of fish size on the TDG supersaturation tolerance of fish, juvenile grass carp (Ctenopharyngodon idellus) of two sizes were subjected to TDG-supersaturated water at high levels (125\%, 130\%, 135\% and 140\%). Furthermore, varying flood flows may cause different TDG levels during the flood season. Fish may be subjected to low, chronic exposure to TDG before peak flooding occurs. However, TDG tolerance in fish subjected to high levels of TDG-supersaturated water after chronic exposure is rare. To further investigate the tolerance of juvenile grass carp subjected to acute exposure after chronic TDG exposure, juvenile grass carp were exposed to high levels of TDG-supersaturated water (125-140\%) after receiving $96 \mathrm{~h}$ of chronic exposure (115\% TDG).
\end{abstract}

Results: In the single acute exposure and multiple exposures (acute exposure after chronic exposure), similar abnormal behaviours and symptoms of gas bubble disease (GBD) were observed in the juvenile grass carp subjected to the TDG-supersaturated water. No abnormal behaviour or mortality was observed in fish in the first chronic exposure of the multiple-exposure treatment. As the TDG level increased, the mortality of the large and small juvenile grass carp increased. The median lethal time $\left(L T_{50}\right)$ for the large juvenile grass carp was 36.55, 21.75 and $6.37 \mathrm{~h}$ at $130 \%, 135 \%$ and 140\% TDG levels, respectively, while the $\mathrm{LT}_{50}$ value of the small juvenile grass carp was $88.13,61.49$ and $35.88 \mathrm{~h}$ at the same TDG levels, respectively. In addition, the $\mathrm{LT}_{50}$ value of juvenile grass carp during acute TDG exposure after chronic exposure was $26.22,7.54$ and $5.34 \mathrm{~h}$ at $130 \%, 135 \%$ and $140 \%$ TDG levels, respectively.

Conclusion: The tolerance of juvenile grass carp decreased with increasing TDG levels. The large juvenile grass carp had weaker tolerance to TDG-supersaturated water than the small juvenile grass carp. In addition, compared with juvenile grass carp subjected to single acute exposure, juvenile grass carp subjected to multiple exposures exhibited lower tolerance and were more vulnerable to the adverse effects of TDG.

Keywords: Total dissolved gas (TDG), Grass carp (Ctenopharyngodon idellus), Gas bubble disease (GBD), Median lethal time $\left(L T_{50}\right)$, Multiple exposures

*Correspondence: Ixq99005@163.com;0120010016@mail.xhu.edu.cn 'Zequn Fan and Yuxuan Deng contributed equally

1 Key Laboratory of Fluid and Power Machinery, Ministry of Education, Xihua University, Chengdu 610039, China

Full list of author information is available at the end of the article

\section{Background}

Since the twentieth century, the construction and operation of high dams have brought some negative effects to downstream river health, especially in China. During the flood season, total dissolved gas (TDG) supersaturation occurs when the gas pressure exceeds atmospheric pressure in the stilling basin downstream of the 
dam. When the TDG level reached a certain value, fish showed marked signs of gas bubble disease (GBD) that threatened their survival [1-3]. Previous researchers described that some endemic fish exhibited GBD symptoms in the Columbia River basin where the TDG levels were distributed within the range of $105-130 \%$ [4]. It has been reported that TDG can cause varied GBD symptoms in fish (e.g., haemorrhages, exophthalmia and excessive bubbles on the fins) and increase mortality $[2,5,6]$. However, compensatory water depth may decrease the damage of TDG to fish and contribute a positive influence on their tolerance [7-9].

In recent years, the problem of TDG supersaturation has attracted increasing attention due to the development of hydropower projects in the Yangtze River [1012]. Many studies have been carried out to determine the effects of TDG on benthic species that inhabit the upper Yangtze River, such as David's schizothoracin Schizothorax davidi, Prenant's schizothoracin Schizothorax prenanti and rock carp Procypris rabaudi. The results of these studies showed that the species exhibited typical GBD signs [13-15]. Different fish exhibited varied tolerances to TDG supersaturation (Rock carp $<$ Prenant's schizothoracin $<$ David's schizothoracin). At present, only limited studies have focused on the effect of TDG on fish inhabiting the middle and lower layers of water [10]. In addition, few studies have assessed the impact of fish size on their tolerance to TDG supersaturation. Grass carp (Ctenopharyngodon idellus), dwelling in the middle and lower layers of water, is one of the four traditional commercial fishes in China. The habitat of grass carp has been destroyed due to the development of high dams. In this paper, grass carp of different sizes were selected to explore the effect of size on TDG supersaturation tolerance.

Furthermore, before flood discharge, the TDG level usually maintains a low value. When peak flooding occurs, a discharged flood may cause a high TDG level in the water downstream of the dam $[16,17]$. Previous studies have suggested that chronic exposure could have negative impacts, such as bladder inflation, immunosuppression and decreased growth [18-21]. Fish dwelling downstream of the dam may be subjected to chronic TDG exposure at a low level prior to peak flooding. However, few studies have mentioned the tolerance of fish subjected to high levels of TDG-supersaturated water after chronic exposure. In this study, we also attempted to further evaluate the effect of TDG supersaturation on the tolerance of juvenile grass carp during acute exposure after chronic exposure. The results may provide important information for the protection of aquatic organism diversity and the operation of hydropower stations.

\section{Materials and methods}

\section{Experimental fish and devices}

Grass carp usually live in the middle and lower layers of water in the river. It is an important economic species in China and has high economic value. In this study, juvenile grass carp were obtained from Meishan Tianhe Fishery Co. Ltd. in Sichuan Province.

The experimental system for generating TDG-supersaturated water was described by Li et al. [22]. The system consisted of water flumes, water tanks, a water pump, a pressure vessel and an air compressor. In this system, compressed air was injected into an autoclave with water from the water flume to produce a high level of TDG-supersaturated water. The water was mixed with $100 \%$ TDG water to obtain varied levels of TDG-supersaturated water. The TDG level was detected by utilizing a Point Four tracker (Point Four Systems, Coquitlam, BC, Canada). A heater was employed to maintain the water temperature. A Multiparameter Water Quality Sonde (YSI 6600, NCL of Wisconsin Inc., USA) was employed to detect the $\mathrm{pH}$, water temperature and dissolved oxygen (DO) level. The weight and fork length of the dead fish were measured by an electronic balance and a ruler, respectively.

\section{Acute lethal experiments}

In this experiment, juvenile grass carp of two sizes were chosen to assess the tolerance of fish subjected to TDG-supersaturated water. Before the experiment, a 720-L water tank (TDG: $100 \%$, temperature: $22 \pm 0.5{ }^{\circ} \mathrm{C}$ (mean \pm SD), DO: $7.1 \pm 0.7 \mathrm{mg} / \mathrm{L}$ and $\mathrm{pH}: 7.7 \pm 0.2$ ) was used to rear juvenile grass carp for 4 days so that the experimental fish could adapt to the new environment. After adaptation, 400 lively juvenile grass carp (200 large juveniles and 200 small juveniles) were selected for acute lethal experiments. Based on the existing survey, the levels of TDG supersaturation downstream of most dams were lower than 140\% [23-25]. TDG supersaturation caused by the flood discharge can remain at a high level over a long distance $(180 \mathrm{~km})$ downstream of dams [26]. Therefore, TDG levels of 100\%, 125\%, 130\%, 135\% and $140 \%$ were set in this study. The control group was $100 \%$ TDG. Each TDG level had duplicate tanks (20 fish per tank (height: $0.6 \mathrm{~m}$; length: $0.6 \mathrm{~m}$; width: $0.6 \mathrm{~m}$; water depth: $0.45 \mathrm{~m}$ )). The acute lethal experiment lasted for $96 \mathrm{~h}$. In the acute lethal experiment, the water quality conditions were consistent with the adaptation conditions, and TDG was measured every hour. The GBD symptoms and abnormal behaviours (e.g., swimming rapidly, jumped up and breathing rapidly) of the fish were observed every $5 \mathrm{~min}$. The time of death, GBD symptoms, weight and fork length of each dead fish were recorded. Experimental fish were deemed to have died 
when they stopped swimming, the gills stopped moving, and they were unresponsive to touch within $60 \mathrm{~s}$.

\section{Acute lethal experiments after chronic exposure}

The size of the juvenile grass carp used in this experiment was similar to that of the large juvenile fish used in the above acute lethal experiment. Before the experiment began, the juvenile grass carp were moved into a 720-L tank (TDG: $100 \%$, temperature: $22.1 \pm 0.7^{\circ} \mathrm{C}$, DO: $7.2 \pm 0.4 \mathrm{mg} / \mathrm{L}$ and $\mathrm{pH}: 7.5 \pm 0.3)$ for 4 days to adapt to the new environment. After adaptation, the juvenile grass carp were placed in a 720-L tank with 115\% TDGsupersaturated water for $96 \mathrm{~h}$. The abnormal behaviours, GBD symptoms and time of death were recorded during chronic exposure. After chronic exposure (96 h), 200 lively juvenile grass carp were selected for acute lethal experiments, and the TDG levels were equal to those in the above acute lethal experiments (100\%, 125\%, 130\%, $135 \%$ and $140 \%$ ). The control group was 100\% TDG. Each TDG level had duplicate tanks (20 fish per tank (height: $0.6 \mathrm{~m}$; length: $0.6 \mathrm{~m}$; width: $0.6 \mathrm{~m}$; water depth: $0.45 \mathrm{~m}$ )). The acute lethal experiments after chronic exposure lasted for $96 \mathrm{~h}$. In this experiment, the water quality conditions were consistent with the adaptation conditions, and TDG was measured every hour. The GBD symptoms and abnormal behaviours (e.g., swimming rapidly, jumping up and breathing rapidly) of the fish were observed every $5 \mathrm{~min}$. The time of death, GBD symptoms, weight and fork length of each dead fish were recorded.

\section{Statistical analysis}

Mortality was an indicator utilized to assess the death process of experimental fish subjected to different levels of TDG-supersaturated water and can be described as follows:

$$
p=\frac{n}{N} \times 100 \%,
$$

where $p$ is the mortality of the experimental fish, $n$ is the number of dead fish, and $N$ is the total number of the experimental fish.

The median lethal time $\left(\mathrm{LT}_{50}\right)$ was considered to determine the tolerance of experimental fish to TDG-supersaturated water. A regression line was fitted through the logarithmic values of the lethal times, and the probability unit value of mortality was calculated as follows:

$$
P(C)=R\left(\mathrm{c}_{e}\right) \times \lg T+J\left(\mathrm{c}_{e}\right),
$$

where $P(C)$ is the probability unit, $R\left(c_{e}\right)$ and $J\left(c_{e}\right)$ are the slope and intercept of the regression line, respectively, $c_{e}$ is the TDG level, and $T$ is the lethal time of the experimental fish. The time was considered the $\mathrm{LT}_{50}$ when the probability unit value was 5 [27].
Differences in the length and weight of the juvenile grass carp of the two sizes and the impact of TDG supersaturation on their tolerances were analysed by one-way analysis of variance (ANOVA). Furthermore, the post hoc multiple comparison test (least significant difference test) was used to determine the differences between the $\mathrm{LT}_{50}$ values of the juvenile grass carp subjected to different levels of TDG-supersaturated water. Tamhane's T2 test was used when there was inhomogeneous variance. Differences were considered significant at $P<0.05$.

\section{Results}

The mean weight and fork length of the large juvenile grass carp were $1.64 \pm 0.24 \mathrm{~g}$ and $5.17 \pm 0.47 \mathrm{~cm}$, respectively. The mean weight and fork length of the small juvenile grass carp were $0.17 \pm 0.03 \mathrm{~g}$ and $2.53 \pm 0.27 \mathrm{~cm}$, respectively. There were significant differences in the weight and fork length between the large and small juveniles (Fig. 1) (ANOVA for weight: $F=580.182$; $d f=1,30$; $p<0.05$. ANOVA for fork length: $F=375.303 ; d f=1,30$; $p<0.05$ ).

\section{Symptoms of GBD in grass carp}

After $1 \mathrm{~h}$ of acute exposure, experimental fish showed abnormal behaviours at the 135\% and 140\% TDG levels.

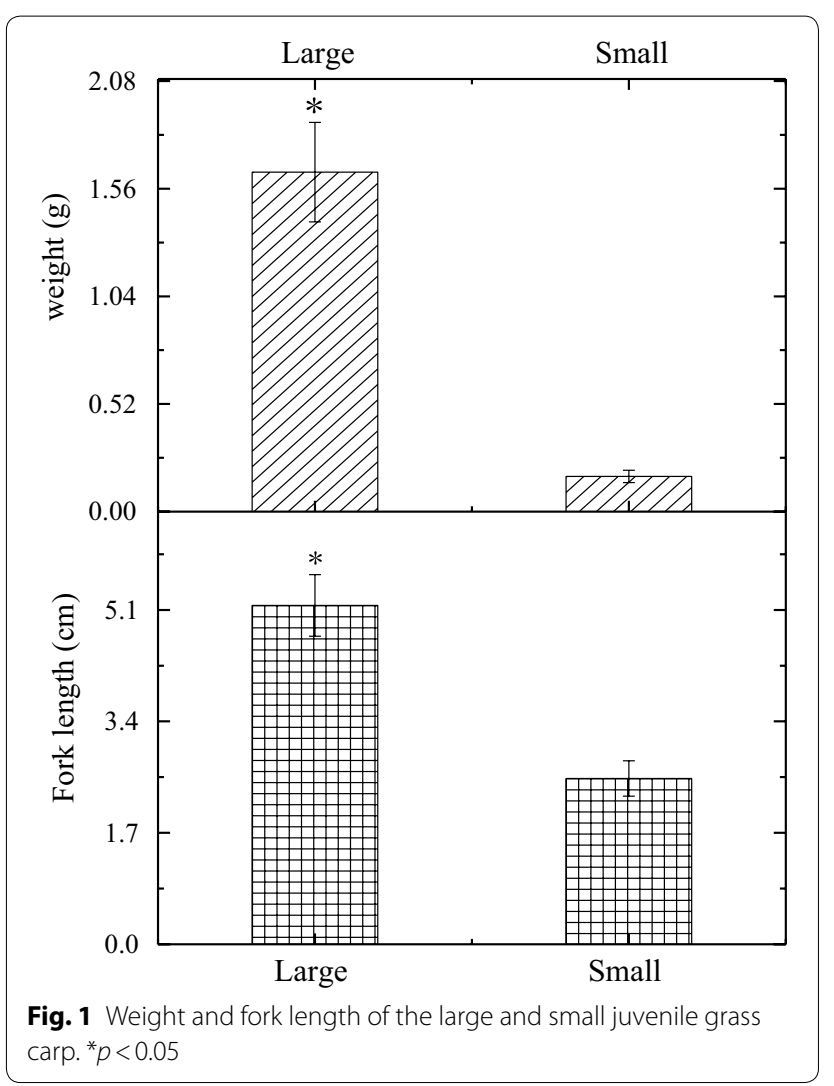


Experimental fish swam erratically with rapid breathing at the water surface. After $1.5 \mathrm{~h}$, fish gradually lost their equilibration and were surrounded by numerous gas bubbles. As the exposure time increased, many fish began to lose their swimming ability (motionless on the water surface, disorientation and loss of equilibrium), and some fish raised their heads out of the water. After $3 \mathrm{~h}$, the fish exposed to $140 \%$ TDG began to die and floated on the water surface. The obvious signs of GBD in dead grass carp were caudal fin bleeding, dorsal fin bubbles, loss of scales, and abdominal swelling (Fig. 2). The above phenomena were also observed in juvenile grass carp at the other TDG levels (125\%, 130\% and 135\%). Juvenile grass carp of the two sizes exhibited similar symptoms of GBD and abnormal behaviours.

\section{Acute lethality experiment}

The relationship between mortality and exposure time in juvenile grass carp is shown in Fig. 3. For the large juvenile grass carp (Fig. 3a), the death of the first fish occurred within $3 \mathrm{~h}$ at the $140 \%$ TDG level, and mortality reached $100 \%$ within $20 \mathrm{~h}$. At 135\% TDG, 100\% mortality was observed after $85 \mathrm{~h}$. At the other TDG levels (125\% and 130\%), the death of the first fish occurred within $10 \mathrm{~h}$. Fourteen and 4 fish survived at the abovementioned supersaturation levels at the end of the experiment. Figure $3 \mathrm{a}$ also shows that the mortality of juvenile fish exceeded $75 \%$ at higher TDG levels ( $\geq 130 \%)$, while the mortality was only $30 \%$ at the $125 \%$ TDG level.

For the small juvenile grass carp, the relationship between mortality and exposure time is shown in Fig. 3b. The death of the first fish occurred within $5 \mathrm{~h}$ at the $140 \%$ TDG level, and the mortality reached $100 \%$ within $96 \mathrm{~h}$. Compared with the large juvenile grass carp, the number of dead small juvenile grass carp declined, and mortality was $62.5 \%$ at the $130 \%$ and $135 \%$ TDG levels at the end of the experiment. After $20 \mathrm{~h}$, the small juvenile grass carp began to die at TDG levels of $125 \%, 130 \%$ and $135 \%$, and the mortalities of small juvenile grass carp were lower (20-40\%) than those of the large juvenile grass carp. The results indicated that the mortality of the large and small juvenile grass carp increased obviously with increased
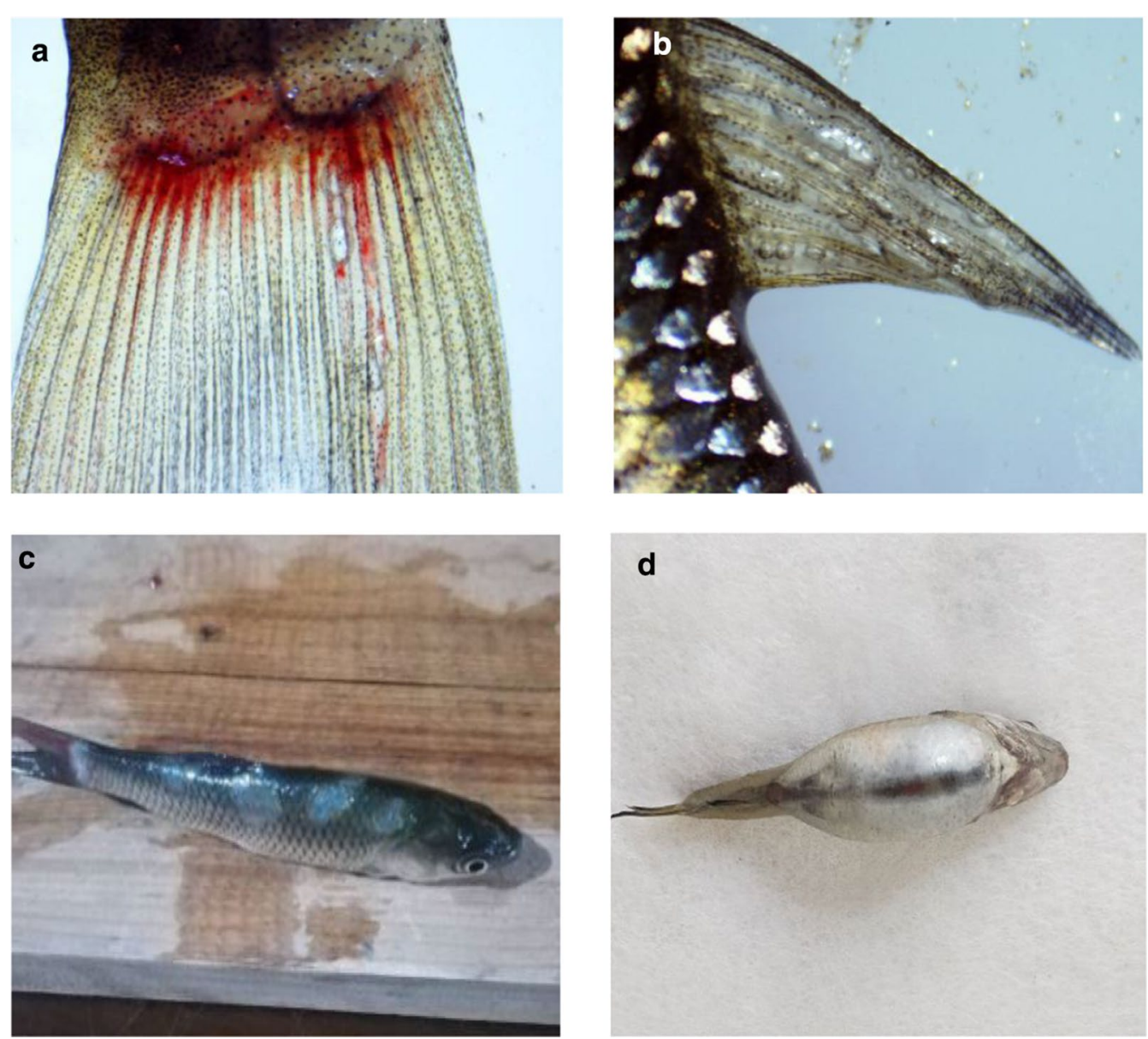

Fig. 2 GBD symptoms of juvenile grass carp: a caudal fin bleeding, b dorsal fin bubbles, $\mathbf{c}$ loss of scales, and $\mathbf{d}$ abdominal swelling 

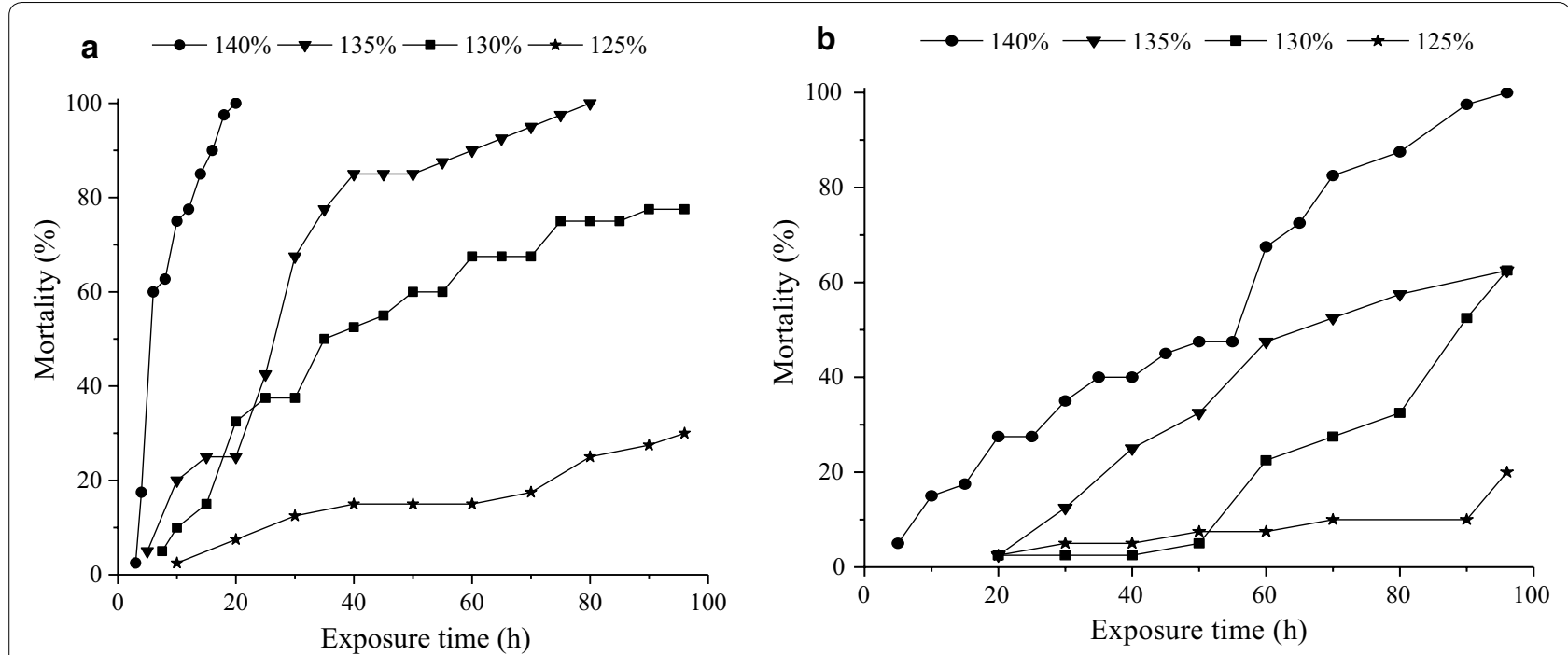

Fig. 3 Mortality of large and small juvenile grass carp subjected to different TDG levels: a large juvenile grass carp; $\mathbf{b}$ small juvenile grass carp

Table 1 The $\mathrm{LT}_{50}$ values of the large and small juvenile grass carp subjected to TDG-supersaturated water

\begin{tabular}{lll}
\hline Experimental groups & TDG levels (\%) & LT $_{\mathbf{5 0}}$ \\
\hline Large juvenile grass carp & 140 & $6.37 \pm 0.45^{\mathrm{A}}$ \\
& 135 & $21.75 \pm 0.87^{\mathrm{B}}$ \\
& 130 & $36.55 \pm 6.95^{\mathrm{C}}$ \\
& 125 & - \\
Small juvenile grass carp & 140 & $35.88 \pm 8.08^{\mathrm{a}}$ \\
& 135 & $61.49 \pm 8.66^{\mathrm{b}}$ \\
& 130 & $88.13 \pm 6.13^{\mathrm{C}}$ \\
& 125 & -
\end{tabular}

The data are described as the mean $\pm \mathrm{SD}(n=2)$. Letters following the $\mathrm{LT}_{50}$ values indicate the results from a post hoc multiple comparison test between varied TDG levels. Capital letters apply to the effects of TDG on the large juvenile grass carp, while lower-case letters apply to the effects of TDG on the small juvenile grass carp. Values that do not share a common letter are significantly different

- indicates that the $\mathrm{LT}_{50}$ value of juvenile grass carp at the $125 \%$ TDG level was not calculated (more than half of the individuals were still alive at the end of the experiment)

exposure time. Throughout the experiment, no fish died in the 100\% TDG-saturated water.

The $\mathrm{LT}_{50}$ values of the juvenile grass carp of different sizes are presented in Table 1 . The $\mathrm{LT}_{50}$ values of the large juvenile grass carp were 36.55, 21.75 and $6.37 \mathrm{~h}$ at $130 \%, 135 \%$ and $140 \%$ TDG, while the $\mathrm{LT}_{50}$ values of the small juvenile grass carp were $88.13,61.49$ and $35.88 \mathrm{~h}$ at the above TDG levels, respectively. The $\mathrm{LT}_{50}$ value of the experimental fish was not calculated at the $125 \%$ TDG level because more than half of the individuals were still alive at the end of the experiment. With decreasing TDG level, the $\mathrm{LT}_{50}$ of the experimental fish

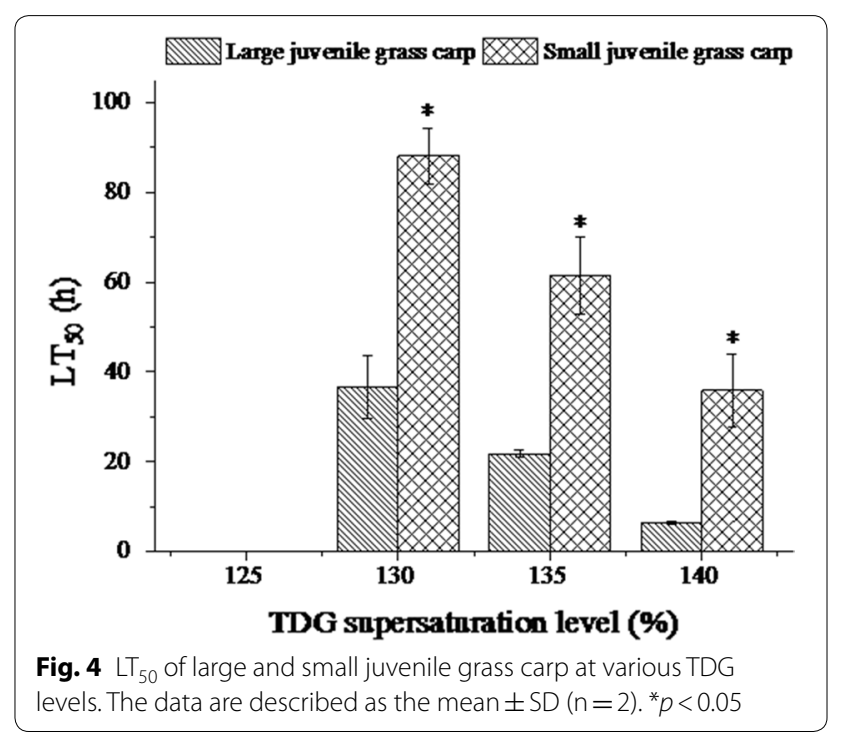

in each group increased significantly (for the large juvenile grass carp: $F=27.721 ; d f=2,3 ; p<0.05$; and for the small juvenile grass carp: $F=23.026$; $d f=2,3 ; p<0.05$ ). For the large and small juvenile grass carp, there were significant differences between the $\mathrm{LT}_{50}$ values of the $130 \%, 135 \%$ and $140 \%$ TDG levels $(p<0.05)$. Furthermore, Fig. 4 also shows that at a given TDG level, the $\mathrm{LT}_{50}$ of the small juvenile grass carp was much higher than that of the large juvenile grass carp, and a significant difference was found between the $\mathrm{LT}_{50}$ values of the large and small juvenile grass carp $(p<0.05)$. The results demonstrated that the large juvenile grass carp 
were more sensitive to TDG than the small juvenile grass carp.

\section{Acute lethal experiments after chronic exposure}

In the first chronic exposure phase, mild GBD symptoms were observed in juvenile grass carp. However, no fish died, and no abnormal behaviour was observed. In the second acute exposure phase after chronic exposure, the GBD symptoms of juvenile grass carp were consistent with those in the acute lethal experiment. Compared with acute exposure, GBD symptoms of juvenile grass carp appeared early with multiple TDG exposures (acute exposure after chronic exposure).

In Fig. 5, at high TDG levels ( $\geq 130 \%)$, the death of the first fish occurred within $5 \mathrm{~h}$, which was earlier than that of large juvenile grass carp during acute exposure $(8 \mathrm{~h})$. At the end of the acute exposure experiment after chronic exposure, the mortalities $(>80 \%)$ were higher than those of the large juvenile grass carp during the single acute exposure experiment (75\%) at the above three TDG levels. The mortality of the juvenile grass carp reached $100 \%$ within 15 and $30 \mathrm{~h}$ at the levels of $140 \%$ and $135 \%$, respectively. At the 130\% TDG level, mortality reached $85 \%$ at $75 \mathrm{~h}$, and no fish died until the end of the experiment. Furthermore, experimental fish began to die at the $125 \%$ TDG level after $20 \mathrm{~h}$, but only $35 \%$ of the fish died at the end of the experiment. Throughout the experiment, no fish died in the 100\% TDG-saturated water.

In Table 2, it can be seen that the $\mathrm{LT}_{50}$ values of the juvenile grass carp increased significantly with decreasing TDG levels in the acute exposure experiment after chronic exposure $(F=20.316 ; d f=2,3 ; P<0.05)$. The $\mathrm{LT}_{50}$ values of juvenile grass carp were $26.22,7.54$ and $5.34 \mathrm{~h}$ at

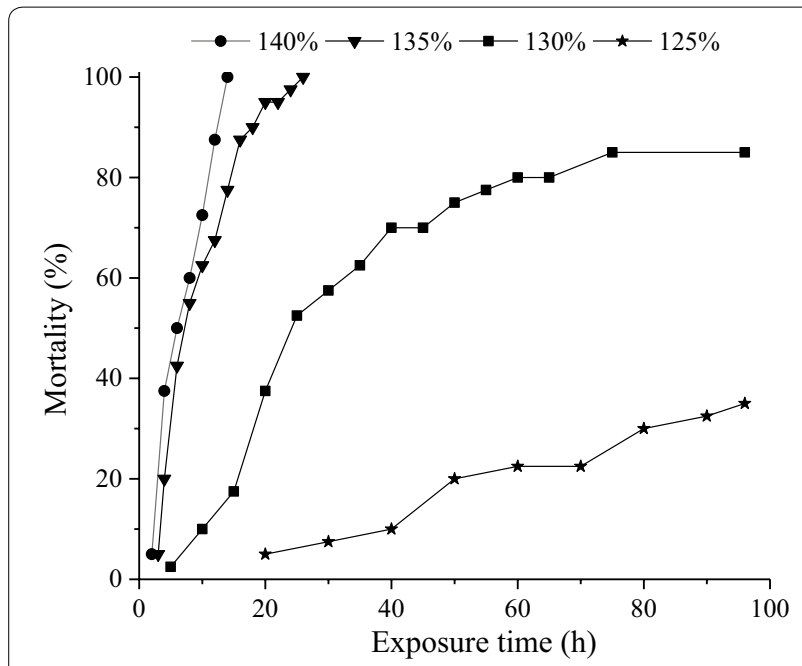

Fig. 5 Mortality of juvenile grass carp subjected to different levels of TDG-supersaturated water after chronic exposure
Table 2 The $\mathbf{L T}_{50}$ values of juvenile grass carp subjected to different levels of TDG-supersaturated water after chronic exposure (multiple exposures)

\begin{tabular}{lll}
\hline Exposure condition & TDG levels (\%) & \multicolumn{1}{c}{$\mathbf{L T}_{\mathbf{5 0}}$} \\
\hline Multiple exposures & 140 & $5.34 \pm 0.42^{\mathrm{a}}$ \\
& 135 & $7.54 \pm 1.65^{\mathrm{ab}}$ \\
& 130 & $26.22 \pm 5.99^{\mathrm{c}}$ \\
& 125 & - \\
\hline
\end{tabular}

The data are described as the mean $\pm S D(n=2)$. Letters following the $\mathrm{LT}_{50}$ values indicate the results from a post hoc multiple comparison test between varied TDG levels. Lower-case letters apply to the effects of TDG on juvenile grass carp. Values that do not share a common letter are significantly different

- indicates that the $\mathrm{LT}_{50}$ value of juvenile grass carp at the 125\% TDG level was not calculated (more than half of the individuals were still alive at the end of the experiment)

the $130 \%, 135 \%$ and $140 \%$ TDG levels, respectively. There was a significant difference between the $\mathrm{LT}_{50}$ values of the $130 \%$ and $135 \%$ levels $(p<0.05)$. However, compared to that of the $140 \%$ TDG level, the $\mathrm{LT}_{50}$ value of the juvenile grass carp did not significantly increase at the $135 \%$ TDG level $(p>0.05)$. The $\mathrm{LT}_{50}$ value of juvenile grass carp was not calculated at the 125\% TDG level because more than half of the individuals survived the experiment.

Furthermore, at the same TDG supersaturation level, the $\mathrm{LT}_{50}$ of juvenile grass carp subjected to a single acute exposure was higher than that of juvenile grass carp subjected to multiple exposures (Fig. 6). There was a significant difference in $\mathrm{LT}_{50}$ values of juvenile grass carp at the $135 \%$ TDG level between the single acute exposure and multiple exposures $(p<0.05)$. However, no significant differences were found for the $\mathrm{LT}_{50}$ values of the $130 \%$ and $140 \%$ TDG levels.

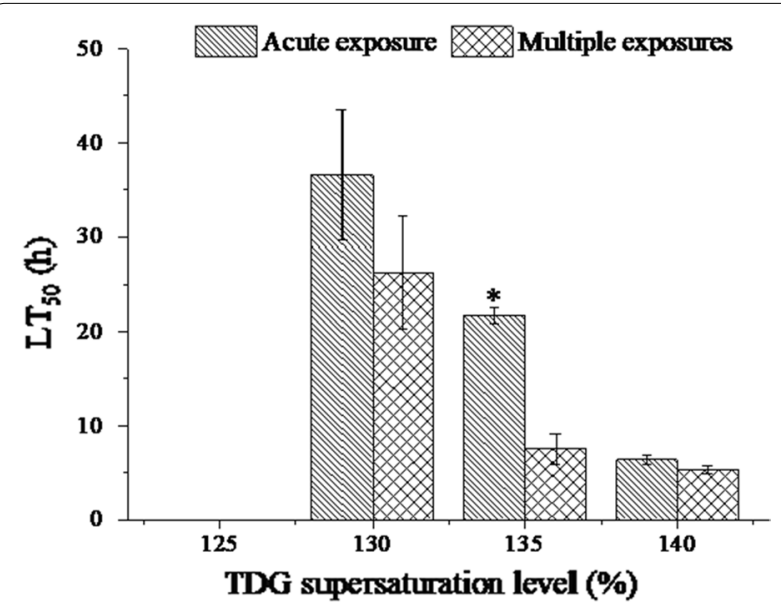

Fig. $6 L T_{50}$ values of the juvenile grass carp subjected to acute exposure and multiple exposures at different TDG levels. The data are described as the mean $\pm S D(n=2) .{ }^{*} p<0.05$ 


\section{Discussion}

Previous studies have demonstrated that GBD symptoms are often found in fish exposed to TDG-supersaturated water [28-31]. Some typical GBD symptoms were described, such as excessive bubbles on the fins, exophthalmia, haemorrhages and swelling of the swimming bladder [6, 22, 32, 33]. In this study, juvenile grass carp subjected to TDG-supersaturated water exhibited caudal fin bleeding, dorsal fin bubbles, loss of scales and abdominal swelling. Furthermore, TDG supersaturation can cause abnormal behaviours in fish, such as faster breathing, loss of the ability to swim and loss of balance [34-36]. The present study showed that similar abnormal behaviours were observed in juvenile grass carp.

It has been illustrated that fish subjected to TDGsupersaturated water would easily die from GBD $[15,37]$. Although GBD produces varied signs and lesions, the cause of death is usually attributed to a lack of oxygen due to blood stasis. The gas in the gill filaments has been described as the most constant and pronounced lesion of GBD. Reduced gas in the vascular system might cause emboli in the gill arterioles [38, 39], leading to death of the fish due to anoxia.

Many studies have indicated that high levels of TDG supersaturation result in the increasing mortality of fish [40, 41]. Ji et al. [42] reported that Prenant's schizothoracin had higher mortality at high TDG levels (130\% and $135 \%)$ than at low TDG levels (110\% and $115 \%)$. Some researchers found that the tolerance of Chinese sucker decreased with increasing TDG levels [22, 43]. In this study, the $\mathrm{LT}_{50}$ values of large and small juvenile grass carp at the 130\% TDG level (36.55 and $88.13 \mathrm{~h}$, respectively) were much higher than those at the $140 \%$ TDG level (6.37 and $35.88 \mathrm{~h}$, respectively) (Table 1). The results showed that the $\mathrm{LT}_{50}$ values of juvenile grass carp decreased significantly with increasing TDG level. Furthermore, related studies have been performed to evaluate the influence of TDG supersaturation on the different species inhabiting the Yangtze River (e.g., Prenant's schizothoracin, Rock carp, Chinese sucker and Silver carp) $[14,15,44,45]$. Table 3 describes the $\mathrm{LT}_{50}$ values of four species and juvenile grass carp at four TDG levels (125\%, $130 \%, 135 \%$ and $140 \%)$. This clearly shows that the juvenile grass carp exhibited higher tolerance than other species in TDG-supersaturated water.

It has been illustrated that size is an important factor in the tolerance of fish subjected to TDG [37, 46]. Smiley et al. [37] indicated that there were obvious differences in the sensitivity and response of white sea bass to TDG-supersaturated water with respect to fish size. The large white sea bass was more sensitive to TDG supersaturation than the small white sea bass. Dawley et al. [46] tested the tolerance of chinook salmon of
Table 3 The $\mathbf{L T}_{50}$ values of different species subjected to TDG-supersaturated water

\begin{tabular}{|c|c|c|c|c|c|}
\hline \multirow[t]{2}{*}{ Fish species } & \multicolumn{5}{|c|}{$\mathrm{LT}_{50}(\mathrm{~h})$} \\
\hline & $125 \%$ & $130 \%$ & $135 \%$ & $140 \%$ & References \\
\hline Prenant's schizothoracin & 9.7 & 8.7 & 8.0 & 4.6 & [10] \\
\hline Rock carp & 10.5 & 5.1 & 4.3 & 3.1 & [11] \\
\hline Chinese sucker & 12.4 & 7.4 & 4.6 & 3.9 & [39] \\
\hline Silver carp & 67.7 & 28.8 & 11.2 & 5.6 & [40] \\
\hline Grass carp & - & 36.55 & 21.75 & 6.37 & Present study \\
\hline
\end{tabular}

- indicates that the $\mathrm{LT}_{50}$ value of fish at the $125 \%$ TDG level was not calculated (more than half of the individuals were still alive)

various sizes to a $112 \%$ total gas pressure. They found that 40 -mm-long fish were more tolerant to TDG supersaturation than fish that were $53 \mathrm{~mm}$ or $67 \mathrm{~mm}$ long. In this study, the $\mathrm{LT}_{50}$ values of the large juvenile grass carp were $36.55,21.75$ and $6.37 \mathrm{~h}$ at the $130 \%$, $135 \%$ and $140 \%$ TDG levels, while the $\mathrm{LT}_{50}$ values of small juvenile grass carp were $88.13,61.49$ and $35.88 \mathrm{~h}$ at the same TDG levels, respectively. This result indicated that juvenile grass carp of different sizes showed varied tolerances, and the large juvenile grass carp had a lower tolerance to TDG-supersaturated water than the small juvenile grass carp. The results of this study were consistent with those of previous studies, and the large juvenile grass carp were more sensitive to TDG than the small juvenile grass carp. We speculated that differences caused by different sizes might be due to differential metabolism, loss of emboli from the blood vessels, and even differences in the target organs due to the different developmental stages of the fish.

Existing studies have demonstrated that chronic exposure to TDG causes oxidative stress and cell damage in rock carp [20]. Yuan et al. [21] pointed out that Leptobotia elongata was exposed to lower TDG levels (110\% and $120 \%)$ for $96 \mathrm{~h}$ and then exposed to higher TDG levels (130\% and 140\%). The time of death of fish exposed to $140 \%$ TDG after exposure to low TDG levels $(110 \%$ and $120 \%)$ was reduced by $41.4 \%$ and $52.05 \%$ compared with that of the single acute exposure (140\%), respectively. The time of death of fish exposed to $130 \%$ TDG after exposure to low TDG levels $(110 \%$ and $120 \%$ ) was reduced by $25.56 \%$ and $75.81 \%$ compared with that of the single acute exposure (130\%), respectively. In this study, the juvenile grass carp did not die in the first chronic exposure phase $(96 \mathrm{~h})$, but they died faster in the subsequent acute exposure experiment than when exposed only acutely. The $\mathrm{LT}_{50}$ values of juvenile grass carp during multiple exposures were $34.67 \%$ and $71.74 \%$ of that of juvenile grass carp during single acute exposure at TDG levels of $135 \%$ and $130 \%$, 
respectively. This result indicated that chronic exposure accelerated the death of fish during acute exposure after chronic exposure. A low TDG level of the supersaturated water had a chronic injury effect on juvenile grass carp. Furthermore, in the single acute exposure and multiple exposures, there was a significant difference in $\mathrm{LT}_{50}$ values at the $135 \%$ TDG level. However, the $\mathrm{LT}_{50}$ value was not significantly different between the $130 \%$ and $140 \%$ TDG levels (Fig. 6). It is possible that chronic exposure did not cause greater damage of organism to the grass carp at TDG levels less than 130\% (multiple exposures). When the TDG level reached a high value (140\%), excessive TDG in the water caused mass mortality of fish due to the rapid emergence of embolisms. Thus, a high TDG level is a fatal factor for juvenile grass carp, and some measures should be taken to minimize the exposure time of grass carp exposed to high TDG levels. Even so, compared with the single acute exposure, acute TDG exposure after chronic exposure (multiple exposures) led to lower $\mathrm{LT}_{50}$ values in juvenile grass carp. Long-term exposure to low TDG levels might pose a great threat to the survival of fish before peak flooding occurs. Limiting the TDG levels to less than $115 \%$ is effective in reducing the effect of long-term exposure of juvenile grass carp to TDG. In addition, long recovery time should be considered for formulating schemes during flood discharge [44]. This possibility should attract attention when formulating reservoir operation schemes and planning protective measures for fish inhabiting downstream of dams.

\section{Conclusions}

This study indicated that increasing TDG levels can decrease the tolerance of juvenile grass carp. Effective measures should be taken to avoid the occurrence of high TDG levels downstream of the dam. Compared with the small juvenile grass carp, the large juvenile grass carp showed lower tolerance to TDG. All juvenile grass carp survived at the $115 \%$ TDG level, and a small number of juvenile grass carp (20-35\%) died at the 125\% TDG level after $96 \mathrm{~h}$. Therefore, the tolerance threshold of juvenile grass carp to TDG-supersaturated water is suggested to be $120 \%$ TDG. In addition, in comparison to grass carp subjected to single acute exposure, grass carp subjected to acute exposure after long-term chronic exposure showed weaker tolerance to TDG and is more vulnerable to the adverse effects of TDG exposure. The results in this study can provide a reference for operational schemes of hydropower plants and the ecological management of rivers in China.

In this study, we only conducted the laboratory experiment to explore the tolerance of juvenile grass carp, the results cannot be directly used to estimate the impact of TDG on juvenile grass carp living in natural rivers. In the future, further field experiments will be performed to investigate the effects of TDG on juvenile grass carp.

\section{Abbreviations}

ANOVA: One-way analysis of variance; ${ }^{\circ} \mathrm{C}$ : Degrees Celsius; $\mathrm{cm}$ : Centimetre; DO: Dissolved oxygen; g: Gram; GBD: Gas bubble disease; h: Hour; L: Litre; LT 50 : The median lethal time; m: Metre; mg/l: Milligram per liter; $\mathrm{pH}$ : Potential of hydrogen; SD: Standard deviation; TDG: Total dissolved gas.

\section{Acknowledgements}

The authors gratefully acknowledge the Key Laboratory of Fluid and Power Machinery at Xihua University for the provision of experimental system for generating TDG-supersaturated water. The authors also thank Chenyang Cao and $\mathrm{Na}$ Li for the valuable discussions on the record and analysis of data.

\section{Authors' contributions}

Conceptualization: ZF and XL. Methodology: CF and QY. Software: YD and LX. Formal analysis: YY and HS. Writing —original draft preparation: ZF. All authors read and approved the final manuscript.

\section{Funding}

This work was supported by the National Natural Science Foundation of China (51509213), Chunhui Plan Project of the Chinese Ministry of Education (191637) and Open Fund Research at the State Key Laboratory of Hydraulics and Mountain River Engineering, Sichuan University, China (Skhl1823).

\section{Availability of data and materials}

The datasets used and analysed during the current study are available from the corresponding author on reasonable request.

\section{Ethics approval and consent to participate}

The experimental proposal was approved by Xihua University. All experiments were executed in accordance with the animal management regulations of Sichuan Province in China.

\section{Consent for publication}

Informed consent was obtained from all individual participants included in the study.

\section{Competing interests}

The authors declared that they have no competing interests.

\section{Author details}

${ }^{1}$ Key Laboratory of Fluid and Power Machinery, Ministry of Education, Xihua University, Chengdu 610039, China. ${ }^{2}$ School of Energy and Power Engineering, Xihua University, Chengdu 610039, China. ${ }^{3}$ State Key Laboratory of Hydraulics and Mountain River Engineering, Sichuan University, Chengdu 610065, China.

Received: 30 January 2020 Accepted: 23 March 2020

Published online: 03 April 2020

\section{References}

1. Ebel WJ (1969) Supersaturation of nitrogen in the Columbia River and its effect on salmon and steelhead trout. Fish Bull 68(1):1-11

2. Meekin TK, Turner BK (1974) Tolerance of salmonid eggs, juveniles and squawfish to supersaturated nitrogen. In: Nitrogen supersaturation investigations in the mid-Columbia River. Washington Department of Fisheries Technical Report, Vol 12. Washington, USA, pp 78126

3. Weitkamp DE, Katz M (1980) A review of dissolved gas supersaturation literature. Trans Am Fish Soc 109:659-702. https://doi.org/10.1577/15488659(1980)109\%3c659:ARODGS\%3e2.0.CO;2

4. May B (1973) Evaluation on the effects of gas bubble disease on fish populations in the Kootenai River below Libby Dam. Proceedings of the 
53rd Annual Conference Western Association of State Fish and Game Commissioners:525540

5. Dawley EM, Ebel WJ (1975) Effects of various concentrations of dissolved atmospheric gas on juvenile chinook salmon and steelhead trout. Fish Bull 73:787-796

6. Jensen JOT, Schnute J, Alderdice DF (1986) Assessing juvenile salmonid responses to gas supersaturation using a general multivariate dose-response model. Can J Fish Aquat Sci 43:1694-1709. https://doi. org/10.1139/f86-213

7. Marsh MC, Gorham FP (1905) The gas disease in fishes. Rep US Bur Fish 1904:343-376

8. Blahm TH, McConnel RJ, Snyder GR (1975) Effect of Gas Supersaturated Columbia River Water on the Survival of Juvenile Salmonids. Final ReportPart 1. National Marine Fisheries Service, Environmental Field Station, Prescott, OR, USA

9. Cao L, Li Y, An RD, Wang YM, Li KF, Buchmann K (2019) Effects of water depth on GBD associated with total dissolved gas supersaturation in Chinese sucker (Myxocyprinus asiaticus) in upper Yangtze River. Sci Rep 9:6828. https://doi.org/10.1038/s41598-019-42971-8

10. Huang X, Li KF, Du J, Li R (2010) Effects of gas supersaturation on lethality and avoidance responses in juvenile rock carp (Procypris rabaudi Tchang). J Zhejiang Univ Sci B 11:806-811. https://doi.org/10.1631/jzus.B1000006

11. Wang YM, Liang R, Tuo Y, Li KF, Hodges B (2015) Tolerance and avoidance behavior towards gas supersaturation in rock carp Procypris rabaudi with a history of previous exposure. N Am J Aquacult 77:478-484. https://doi. org/10.1080/15222055.2015.1059913

12. Xue SD, Wang YM, Li KF, Liang RF, Li Y, Feng JJ, Cao L (2019) In situ study on the impact of total dissolved gas supersaturation on endemic fish in the Upper Yangtze River. River Res Applic 35:1511-1519. https://doi. org/10.1002/rra.3503

13. Liang RF, Li B, Li KF, Tuo YC (2013) Effect of total dissolved gas supersaturated water on early life of David's schizothoracin (Schizothorax davidi). J Zhejiang Univ Sci B 14:632-639. https://doi.org/10.1631/jzus.B1200364

14. Wang YM, Li KF, Li J, Li R, Deng Y (2015) Tolerance and avoidance characteristics of prenant's schizothoracin Schizothorax prenanti to total dissolved gas supersaturated water. N Am J Fish Manag 35:827-834. https ://doi.org/10.1080/02755947.2015.1052160

15. Xue SD, Wang YM, Liang RF, Li KF, Li R (2019) Effects of total dissolved gas supersaturation in fish of different sizes and species. Int J Env Res Pub He 16:2444. https://doi.org/10.3390/ijerph16132444

16. Tan DC, Ni ZH, Zheng YH, Li LY, Li YF (2006) Dissolved gas supersaturation downstream of dam and its effects on fish. Freshw Fish 36:56-59 (in Chinese)

17. Cheng XJ, Chen YC (2007) Theoretical analysis of dissolved gas supersaturation downstream of sluicing dam and application. Adv Water Sci 18:346. https://doi.org/10.14042/j.cnki.32.1309.2007.03.006

18. Colt J, Orwicz K, Brooks D (1985) The effect of gas supersaturation on the growth of juvenile channel catfish, Ictalurus punctatus. Aquaculture 50:153-160. https://doi.org/10.1016/0044-8486(85)90161-9

19. Shrimpton JM, Randall DJ, Fidler LE (1990) Assessing the effects of positive buoyancy on rainbow trout (Oncorhynchus mykiss) held in gas supersaturated water. Can J Zool 68:969-973. https://doi.org/10.1139/ z90-139

20. Liu XQ, Li KF, Du J, Li J, Li R (2011) Growth rate, catalase and superoxide dismutase activities in rock carp (Procypris rabaudi Tchang) exposed to supersaturated total dissolved gas. J Zhejiang Univ Sci B 12:909-914. https://doi.org/10.1631/jzus.B1100071

21. Yuan Y, Yuan Q, Wang YM, An RD, Li KF (2017) Acute and chronic lethality of total dissolved gas supersaturated water on Leptobotia elongata. Adv Eng Sci 49:56-61. https://doi.org/10.15961/j.jsuese.201600739 (in Chinese)

22. Li N, Fu CH, Zhang J, Liu XQ et al (2019) Hatching rate of Chinese sucker (Myxocyprinus asiaticus Bleeker) eggs exposed to total dissolved gas (TDG) supersaturation and the tolerance of juveniles to the interaction of TDG supersaturation and suspended sediment. Aquac Res 50:1876-1884. https://doi.org/10.1111/are.14071

23. Li R, Li J, Li KF, Deng Y, Feng JJ (2009) Prediction for supersaturated total dissolved gas in high-dam hydropower projects. Sci China Ser E 52:3661-3667. https://doi.org/10.1007/s11431-009-0337-4
24. Qu L, Li R, Li J, Li KF, Deng Y (2011) Field observation of total dissolved gas supersaturation of high-dams. Sci China Ser E 54:156-162. https://doi. org/10.1007/s11431-010-4217-8

25. Feng JJ, Li R, Liang RF, Shen X (2014) Eco-environmentally friendly operational regulation: an effective strategy to diminish the TDG supersaturation of reservoirs. Hydrol Earth Syst Sc 18:1213-1223. https://doi. org/10.5194/hess-18-1213-2014

26. Feng JJ, Li R, Li KF, Li J, Qu L (2010) Study on release process of supersaturated total dissolved gas downstream of high dam. J Hydroelectric Eng 29:7-12 (In Chinese)

27. Shetty AJ, Alwar MC (2007) Acute toxicity studies and determination of median lethal dose. Curr Sci 93(7):917-920

28. Ebel WJ, Raymond HL (1976) Effect of atmospheric gas supersaturation on salmon and steelhead trout of the snake and Columbia rivers. Marin Fish Rev 387:1-14

29. Counihan TD, Miller Al, Mesa MG, Parsely MJ (1998) The effects of dissolved gas supersaturation on white sturgeon larvae. T Am Fish Soc 127:316-322. https://doi.org/10.1577/1548-8659(1998)127\%3c031 6:TEODGS\%3e2.0.CO;2

30. Mesa MG, Weiland LK, Maule AG (2000) Progression and severity of gas bubble trauma in juvenile salmonids. T Am Fish Soc 129:174-185. https:// doi.org/10.1577/1548-8659(2000)129\%3c0174:PASOGB\%3e2.0.CO;2

31. Feng CX, Li N, Wang YM, Liu XQ et al (2019) Effects of total dissolved gas supersaturated water at varying suspended sediment concentrations on the survival of rock carp Procypris rabaudi. Fish Sci 85:1067-1075. https:// doi.org/10.1007/s12562-019-01344-w

32. Stroud RK, Bouck GR, Nebeker AV (1975) Pathology of acute and chronic exposure of salmonid fishes to supersaturated water. In: Adams WA (ed) Chemistry and physics of aqueous gas solutions. The Electrochemical Society, Princeton, pp 435-449

33. Beeman JW, Venditti DA, Morris RG, Gadomski DM et al (2003) Gas bubble disease in resident fish below grand coulee dam: final report of research. USBR, Boise

34. Wyatt EJ, Beiningen KT (1971) Nitrogen gas bubble disease related to a hatchery water supply from the fore bay of a high head re-regulating dam. Fish Commission of Oregon, Portland

35. Krise WF (1993) Effects of one-year exposures to gas supersaturation on lake trout. Prog Fish Cult 55:169-176. https://doi.org/10.1577/15488640(1993)055\%3c0169:eooyet\%3e2.3.co;2

36. Wang YM, An RD, Li Y, Li KF (2017) Swimming performance of rock carp Procypris rabaudi and Prenant's schizothoracin Schizothorax prenanti acclimated to total dissolved gas supersaturated water. N Am J Fish Manag 37:827-834. https://doi.org/10.1080/02755947.2017.1353558

37. Smiley JE, Drawbridge MA, Okiniro MS, Kaufmann RS (2011) Acute effects of gas supersaturation on juvenile cultured white Seabass. T Am Fish Soc 140:1269-1276. https://doi.org/10.1080/00028487.2011.618359

38. Woodbury LA (1942) A sudden mortality of fishes accompanying a supersaturation of oxygen in Lake Waubesa, Wisconsin. T Am Fish Soc 71:112-117. https://doi.org/10.1577/1548-8659(1941)71\%5b112:ASMOF $\mathrm{A} \% 5 \mathrm{~d} 2.0 . \mathrm{CO} ; 2$

39. Renfro WC (1963) Gas-bubble mortality of fishes in Galveston Bay, Texas. T Am Fish Soc 92:320-322. https://doi.org/10.1577/15488659(1963)92\%5b320:GMOFIG\%5d2.0.CO;2

40. Rucker RR, KangaS PH (1974) Effect of nitrogens supersaturated water on coho and chinook salmon. Prog Fish Cult 36:152-156. https://doi. org/10.1577/1548-8659(1974)36\%5b152:EONSWO\%5d2.0.CO;2

41. Weitkamp DE, Katz M (1980) A review of dissolved gas supersaturation literature. T Am Fish Soc 109:659-702. https://doi.org/10.1577/15488659(1980) 109\%3c659:ARODGS\%3e2.0.CO;2

42. Ji QF, Xue SD, Yuan Q, Yuan Y et al (2019) The Tolerance characteristics of resident fish in the upper Yangtze River under varying gas supersaturation. Int J Environ Res Public Health 16:2021. https://doi.org/10.3390/ijerp h16112021

43. Chen SC, Liu XQ, Jiang W, Li KF et al (2012) Effects of total dissolved gas supersaturated water on lethality and catalase activity of chinese sucker (Myxocyprinus asiaticus Bleeker). J Zhejiang Univ Sci B 13:791-796. https:// doi.org/10.1631/jzus.B1200022

44. Cao L, Li KF, Liang RF, Chen SC et al (2015) The tolerance threshold of Chinese sucker to total dissolved gas supersaturation. Aquac Res 47:2804-2813. https://doi.org/10.1111/are.12730 
45. Cao L, Liang RF, Tuo YC, Li Y, Li KF (2016) Influence of total dissolved gassupersaturated water on silver carp (Hypophthalmichthys molitrix). WSE 9:324-328. https://doi.org/10.1016/j.wse.2017.01.001

46. Dawley EM, Schiewe M, Monk B (1976) Effects of long-term exposure to supersaturation of dissolved atmospheric gases on juvenile chinook salmon and steelhead trout in deep and shallow tank tests. pp 110

\section{Publisher's Note}

Springer Nature remains neutral with regard to jurisdictional claims in published maps and institutional affiliations.

\section{Submit your manuscript to a SpringerOpen ${ }^{\odot}$ journal and benefit from:}

- Convenient online submission

- Rigorous peer review

- Open access: articles freely available online

- High visibility within the field

- Retaining the copyright to your article

Submit your next manuscript at $\boldsymbol{\nabla}$ springeropen.com 